\title{
Biomechanical factors in Finite Element Analysis of abdominal aortic aneurysms
}

\author{
Zuzanna Domagala', Hubert Stepak², Pawel Drapikowski ${ }^{3}$, Lukasz Dzieciuchowicz ${ }^{2}$, \\ Malgorzata Pyda ${ }^{4}$, Katarzyna Karmelita-Katulska ${ }^{5}$, Grzegorz Oszkinis ${ }^{2}$ \\ 'Poznan University of Technology, Poland \\ ${ }^{2}$ Department of General and Vascular Surgery, Poznan University of Medical Sciences, Poland \\ ${ }^{3}$ Institute of Control and Information Engineering, Faculty of Electrical Engineering, Poznan University of Technology, Poland \\ ${ }^{4} \mathrm{I}^{\text {st }}$ Clinic of Cardiology, Department of Cardiology, Poznan University of Medical Sciences, Poland \\ ${ }^{5}$ Department of General Radiology, Poznan University of Medical Sciences, Poland
}

\begin{abstract}
The abdominal aortic aneurysm is tenth most common cause of death in Western countries. Since maximal transverse diameter as indication for surgical interventions is often criticized, biomechanics of the aneurysm has been studied to develop new criteria for a treatment. The Finite Element Method is being utilized to predict vessel stability. Computer simulations are proven to have high accuracy of rupture risk assessment, although the impact of all incorporated factors is still not fully known. The objective of this paper is to review the most commonly used biomechanical components of computer analysis, including geometry of the vessel, mechanical properties of the wall, thrombus and calcification, their impact on rupture risk, and methods of modelling blood pressure. Comprehension and precise assessment of biomechanics of aneurysm in terms of Finite Element Analysis have high potential in clinical management of abdominal aortic aneurysm.
\end{abstract}

Key words: Finite Element Analysis, abdominal aortic aneurysm (AAA), biomechanics of abdominal aortic aneurysm (AAA), computer simulation

Acta Angiol 2016; 22, 4: |64-17|

\section{Introduction}

Abdominal aortic aneurysm (AAA) is defined as widening of infrarenal aorta more than $30 \mathrm{~mm}$. It has been proven that there is a positive association between current or past smoking, male gender, age, atherosclerosis, hypertension, family history of AAA or other large arteries aneurysm with aneurysm formation [I]. The natural history of AAA is progressive dilation, which may lead to potentially fatal rupture. The AAA is the $10^{\text {th }}$ cause of death in the Western population. Traditional surgical repair and minimally invasive endovascular repair are the invasive treatment options. Thus, it is crucial to take into account the perioperative complications [2].
Most widely accepted indication for intervention is based on AAA maximum transverse diameter being larger than $5.5 \mathrm{~cm}$. This criterion undergoes discussions [3], since $10-24 \%$ of aneurysms which ruptured are $5.0 \mathrm{~cm}$ or less in diameter [4-6]. Aortic diameters are the cornerstone of current clinical indications for surgery in aortic aneurysms, and some limitations in accuracy measurements may generate errors in clinical decision making. Better understanding, beyond the simple clinical application of diameters, of new biomechanical information may provide a more reliable prediction of the risk of the aneurysm rupture. Hence, individualized, biomechanical approach to treatment of AAA is being studied. Computer simulations with Finite Element 
Method (FEM) is well known engineering tool to assess the probability of mechanical failure of buildings, planes, bridges, etc. This method takes into account geometrical as well as mechanical properties of the investigated object. Since FEM orientated simulation is highly reliable for man-made structures, the examination of biological objects using the same approach seems to be reasonable. However, exact biomechanical factors of aneurysms are difficult to obtain, as it would require harvesting samples of AAA for every patient. That is why some simplifications have been introduced to FEM analysis. The simple geometric measures such as aneurysm volume, maximal wall dilation, ratio of greatest antero-posterior diameter to transverse diameter and curvature have been studied in order to determine the new rupture predictors $[7,8]$. Due to the failure to anticipate the accurate stress distribution, such simplifications were rejected. There is still no consensus regarding the impact of the factors included into computer simulations; however, general guidelines have been outlined.

The aim of this paper is to review biomechanical factors which are often being incorporated into Finite Element Analysis of the AAA. It will help to fully comprehend computer analysis of AAA as a new tool for estimation of the AAA rupture risk.

\section{Biomechanical factors of rupture risk assessment}

Recently, understanding of biomechanics of the abdominal aorta and the AAA has significantly increased. It is due to employment of the engineering tool - FEM into rupture risk assessment. This approach requires several biomechanical information to properly model real behaviour of the AAA. Three factors play crucial role in AAA assessment:geometry of the vessel, mechanical properties of wall, and applied loading from blood stream.

\section{Geometry of abdominal aortic aneurysm}

\section{Wall thickness}

The thickness of physiological abdominal aorta amounts to $1.39 \mathrm{~mm}$ [9]. Wall thickness of AAA is changed due to the dilation of the vessel and possible inflammation or other pathological remodelling. Moreover, the wall thickness is not uniform for whole aneurysm. Since 3D models of the AAA are mostly founded on contrast-enhanced CT scans, the precise wall thickness is not yet possible to observe. As a certain value of this parameter must be assumed, numerous measurements have been conducted to estimate the average AAA wall thickness. The measurements of specimens are given in Table I, whereas the commonly used values of the approximated wall thickness are gathered in Table 2.
Table I. Experimental measurements of wall thickness

\begin{tabular}{|l|c|c|}
\hline Research & \multicolumn{1}{c}{$\begin{array}{c}\text { Range of } \\
\text { measured wall } \\
\text { thickness [mm] }\end{array}$} & $\begin{array}{c}\text { Average wall } \\
\text { thickness } \\
\text { [mm] }\end{array}$ \\
\hline Di Martino et al. [I0] & $2.5-3.6$ & 3.05 \\
\hline Raghavan et al. [I I] & $0.23-4.26$ & 2.25 \\
\hline Reeps et al. [I 2] & $0.85-3.20$ & 2.03 \\
\hline
\end{tabular}

Table 2. Commonly assumed wall thickness in FEM analysis

\begin{tabular}{|l|c|}
\hline Research & $\begin{array}{c}\text { Assumed wall } \\
\text { thickness [mm] }\end{array}$ \\
\hline Mohammad et al. [13] & 1.0 \\
\hline Raut et al. [14] & 1.5 \\
\hline Raghavan et al. [15] & 1.9 \\
\hline Doyle et al. [16] & 2.0 \\
\hline
\end{tabular}

It has been shown that the ruptured aneurysms have significantly thicker wall than electively repaired [10]. The calcified part of the vessel feature great wall thickness in contrast to ruptured regions and both the posterior and right part of AAA is slightly thinner than the rest of the aneurysm [ $\mathrm{I} \mathrm{I}]$. Wall thickness increases in patients suffering from diabetes mellitus, while in cases of chronic kidney disease it is considerably thinner [12].Due to such variations in wall thickness it is important to develop more precise imaging techniques in order to accurately extract vessel shape from AAA scans, as this is crucial factor for reliable FEM analysis.

\section{Presence of intraluminal thrombus and calcifications in AAA wall}

Early FEM analyses of AAA behaviour did not include presence of intraluminal thrombus (ILT) or calcifications $[17,18]$, although those inclusions alter the stress distribution in aneurysm wall. The impact of those structures on the stability of the vessel was a subject of discussion. Some researchers claim that ILT lowers the rupture risk. This purely mechanical standpoint is derived from computer analysis and assumes that additional layer in AAA has protective effect of reducing the wall stress [19-22].

Others underline the degeneration process that occurs in wall due to hypoxia, and ultimately weakens of the tissue [23, 24].This biochemical approach is based on in vivo experiments and tries to capture the micro-changes in wall formation, which could locally affect its strength. Aforementioned transformation includes: the inflammation and neovascularization [25], proteolytic activity [26] - especially increase in elastase production [27], decrease in collagen synthesis [28-30] and production of abnormal collagen [29]. From the clinical point of view, rapid growth and volume of ILT is 
correlated with the high risk of rupture [3I]. This observation confirms that the presence of the thick thrombus at leasthas negative impact on AAA wall strength.

Calcifications are well visible on CT scans. However, from the image processing point of view they could be easily taken as part of the lumen of the AAA as the brightness of contrast enhanced lumen and calcification is similar. The automatic methods of segmentation do not discriminate accurately between adjacent areas of the same level of brightness, which leads to misinterpretation of a lumen geometry. That is why there is a little research which takes into account calcifications in $3 \mathrm{D}$ models of the aneurysm. The unquestionable issue is that this kind of inclusion introduces changes in local wall stress distribution. Although, there is a lack of agreement on how calcifications actually affect it. Since the discussed constituent is stiffer than the rest of the wall, it presents possible stress concentration which is consistent with higher rupture probability $[32,33]$. On the other hand, calcifications cause local wall thickening [ II ] and demonstrate significant load-bearing effects, which results in lowering the wall stress [34].

Since both ILT and calcification alter local mechanical parameters of the wall, it is important to incorporate those pathologies into 3D models of $A A A$ in order to obtain reliable results of FEM analysis.

\section{Shape of AAA}

Although shape of the aneurysm is not a changeable parameter in FEM analysis, since it is retrieved from $\mathrm{CT} / \mathrm{MRI}$ scans, it is important to comprehend its impact on stress distribution. Aortic tortuosity is described as displacement of the lumen position on the consecutive scans. The greater the displacement, the bigger the aneurysm tortuosity. The cross-sectional diameter asymmetry is based on diameter length differences in longitudinal and transverse directions on a single scan.
It has been shown that less tortuous aneurysm with high cross-sectional diameter asymmetry are associated with ruptured AAA [35]. The high wall stress is concentrated in the inflection point of aneurysm geometry, which is defined as a point where the surface changes its shape from concave to convex [36]. Aforementioned points are impossible to observe on $2 \mathrm{D} C T / M R I$ scans, thus the 3D model incorporated into FEM analysis is superior as a rupture location predictor.

\section{Mechanical properties of AAA}

\section{Wall properties}

Reliable FEM analysis of AAA requires mechanical information on wall behaviour. The wall material is widely acknowledged to be hyperelastic [37], which means it has a little to no change in volume when stretching occurs. It can also undergo large deformation and has ability to return to normal shape after stress is removed. Although aneurysm tissue has non-elastic response to stress, some researchers exploit the simplified elastic wall model in order to examine the impact of a single factor on the stress distribution [38-39]. Early works on the wall mechanics employed the isotropic material for an aneurysm [40-4I]. That is, the vessel was assumed to have exactly the same properties (e.g. ability to stretch) in both longitudinal and circumferential direction. Introducing biaxial experiments into investigation of AAA biomechanics revealed the anisotropy of the wall [42]. It turns out that especially circumferential direction is prone to stiffening [43]. The most frequently used wall parameters are gathered in Table 3.

\section{Intraluminal thrombus and calcification properties}

There have been several experiments conducted on AAA specimens in order to determine the mechanical properties of ILT and calcifications.

Table 3. Mechanical parameters of the wall

\begin{tabular}{|c|c|c|}
\hline Research & Features & Mathematical parameters \\
\hline $\begin{array}{l}\text { Raghavan et al. [37] } \\
\text { Wang et al. [44] }\end{array}$ & $\begin{array}{l}\text { Homogeneous } \\
\text { Hyperelastic } \\
\text { Isotropic } \\
\text { Incompressible }\end{array}$ & $\begin{aligned} W & =\alpha\left(I_{B}-3\right)+\beta\left(I_{B}-3\right)^{2} \\
\alpha & =0.174 \mathrm{MPa}, \beta=1.88 \mathrm{MPa}\end{aligned}$ \\
\hline Thubrikar et al. [38] & $\begin{array}{l}\text { Homogeneous } \\
\text { Linear elastic } \\
\text { Isotropic } \\
\text { Incompressible } \\
\end{array}$ & $\begin{array}{c}E=4.66 \mathrm{~N} / \mathrm{mm}^{2} \\
v=0.49\end{array}$ \\
\hline O'Leary at al. [42] & $\begin{array}{l}\text { Homogeneous } \\
\text { Hyperelastic } \\
\text { Anisotropic } \\
\text { Incompressible }\end{array}$ & $\begin{array}{l}W=b_{0}\left[e^{b_{1} \frac{1}{2}\left(\frac{\lambda_{\theta}^{2}-1}{2}\right)^{2}}+e^{b_{2} \frac{1}{2}\left(\frac{\lambda_{L}^{2}-1}{2}\right)^{2}}+e^{b_{3}\left(\frac{\lambda_{\theta}^{2}-1}{2}\right)\left(\frac{\lambda_{L}^{2}-1}{2}\right)}\right] \\
\mathrm{b}_{0}=0.44 \mathrm{~b}_{1}=306.83 \mathrm{~b}_{2}=213.34 \mathrm{~b}_{3}=2|8.7|\end{array}$ \\
\hline
\end{tabular}

$W$ is the strain energy density function and $I_{B}$ stands for the first invariant of Left Cauchy-Green tensor. $E$ stands for Young modulus and $v$ for Poisson's ratio. $\lambda$ is a stretch ratio evaluated in the circumferential $(\phi)$ and longitudinal $(L)$ directions. $\alpha, \beta, b_{0}, b_{1}, b_{2}, b_{3}$ are material coefficients 
Thrombus can be considered as homogeneous or three-layered structure. In both cases there is assumption on linear elasticity, isotropy and incompressibility. However the heterogeneous model is not suitable for FEM analysis since one of the ILT layers is too fragile to be mechanically tested [44]. Raghavan and Wang presented thrombus as hyperelastic material $[20,37]$ whereas Van Dam points out the viscoelastic (both viscous and elastic)behaviour of ILT [45]. It means that discussed inclusion is able to return to former shape after removing the load (elastic property) but it does not occur instantly. Deformation in viscoelastic substances depend on the time and the temperature which are typical viscous characteristics. Finally, the porous nature of ILT was applied in FEM analysis [46, 47]. Porous materials are heterogeneous and consist of two phases: solid and liquid/gas. Each phase alone is homogenous, incompressible and is assumed to be isotropic. The solid phase might be considered as linear elastic (poroelastic) [47] or hyperelastic (porohyperelastic) [46]. The solid material forms a three-dimensional net in voids of which the liquid or gas is present. This model of thrombus highlights the fact that there are blood leakages from the lumen to the AAA wall through ILT. Although each phase separately is incompressible, the assembling of both results in a compressible structure. Porous description of ILT mitigates its protective effect on the wall during computer simulation.

Local wall calcification is present in great number of aneurysms. It consists mostly of elements such as calcium, phosphorus, magnesium, sodium, chloride, potassium sulphur, all in both organic and inorganic compounds [48]. The mechanical properties of the calcified deposits are similar to the bone tissue.Therefore, assumption of incompressibility, linear elasticity, isotropy and homogeneity is valid for all endurance experiments. There are however major differences in values of Young's modulus (Tab. 5). The lowest parameters were obtained for calcified non-aneurysmatic aortic tissue [49]. Due to the lack of information on calcification properties of aneurysm, this value was used in FEM analysis. The following experiments were conducted on proper AAA specimens. However, the results depend on the composition and the degree of calcification. Maier's study covers tissue samples from slightly to highly calcified, thus the range of values was presented [34].

Material information on both constituents is listed in Table 4 and Table 5.

Table 4. Mechanical parameters of ILT

\begin{tabular}{|c|c|c|}
\hline Research & Features & Mathematical parameters \\
\hline Di Martino et al. [50] & $\begin{array}{l}\text { Homogeneous } \\
\text { Linear elastic } \\
\text { Isotropic } \\
\text { Incompressible }\end{array}$ & $\begin{array}{c}E=0.1 I \mathrm{MPa} \\
\mathrm{v}=0.45\end{array}$ \\
\hline Wang et al. [44] & $\begin{array}{l}\text { Heterogeneous } \\
\text { Linear elastic } \\
\text { Isotropic } \\
\text { Incompressible } \\
\end{array}$ & $\begin{array}{l}\text { Luminal layer } \mathrm{E}=0.54 \mathrm{MPa} \\
\text { Medial layer } \mathrm{E}=0.28 \mathrm{MPa} \\
\text { Abluminal layer }- \text { no data }\end{array}$ \\
\hline Vande Geest et al. [5I] & $\begin{array}{l}\text { Homogenous } \\
\text { Hyperelastic } \\
\text { Isotropic } \\
\text { Incompressible }\end{array}$ & $\begin{array}{l}W=\alpha\left(I_{B}-3\right)+\beta\left(I_{B}-3\right)^{2} \\
\alpha=7.98 \mathrm{~N} / \mathrm{cm}^{2}, \beta=8.71 \mathrm{~N} / \mathrm{cm}^{2}\end{array}$ \\
\hline Van Dan et al. [45] & $\begin{array}{l}\text { Homogenous } \\
\text { Viscoelastic } \\
\text { Isotropic } \\
\text { Incompressible }\end{array}$ & $\begin{array}{r}G^{\prime}=\sum_{i=1}^{n} G_{i} \frac{\lambda_{i} \omega}{1+\lambda_{i}^{2} \omega^{2}} \\
\omega=<\mathrm{Irad} / \mathrm{s}(0.16 \mathrm{~Hz}) \\
100 \mathrm{rad} / \mathrm{s}(16 \mathrm{~Hz})>\mathrm{n}=3\end{array}$ \\
\hline Ayyalasomayajula et al. [46] & $\begin{array}{l}\text { Porohyperelastic } \\
\text { Solid part: } \\
\text { homogeneous } \\
\text { hyperelastic } \\
\text { isotropic } \\
\text { incompressible } \\
\text { Fluid part: } \\
\text { homogeneous } \\
\text { isotropic } \\
\text { incompressible }\end{array}$ & $\begin{array}{l}\qquad \sigma_{i j}^{\text {total }}=\sigma_{i j}^{e f f}-p^{f} \delta_{i j} \\
\text { Detailed information on porohyperelastic ILT } \\
\text { is out of scope of this paper and can be found } \\
\text { in [46] }\end{array}$ \\
\hline
\end{tabular}

$E$ stands for Young modulus and $v$ for Poisson's ratio. $W$ is the strain energy density function and $\mathrm{I}_{\mathrm{B}}$ stands for the first invariant of Left Cauchy-Green tensor. $\alpha, \beta$ are material coefficients. G' is the storage modulus. $\lambda_{i}$ and $G_{i}$ are a set of the relaxation time and shear modulus for the ith mode and $\omega$ is angular frequency. $\delta_{i j}^{\text {totol }}$ stands for total stress tensor, $p^{f}$ is the pressure exerted by the pore fluid on the surrounding solid and $\delta_{i j}^{\text {eff }}$ is the effective stress in the solid component. $\delta_{i j}$ is the Kronecker delta [46] 
Table 5. Mechanical parameters of calcification

\begin{tabular}{|c|c|}
\hline Research & Mathematical parameters \\
\hline Speelman et al. [32] & $\begin{array}{c}E=1,47 \mathrm{MPa} \\
\mathrm{v}=0,45\end{array}$ \\
\hline Maier et al. [34] & $\begin{array}{c}\mathrm{E}=<40 \mathrm{MPa}, 450 \mathrm{MPa}> \\
\mathrm{v}=0.4\end{array}$ \\
\hline Marra et al. [48] & $\begin{array}{c}E=20.5 \mathrm{GPa} \\
v=0.4\end{array}$ \\
\hline
\end{tabular}

E stands for Young modulus and $v$ for Poisson's ratio

\section{Loadings}

\section{Static and dynamic impact of blood on AAA}

The impact of blood as a loading force was firstly used as uniform pressure applied to the inner surface of the AAA models. There are several slightly different approaches in that matter. As the CT scans are widely used for extraction of aneurysm shape, certain imaging conditions have to be ensured. It is important to employ ECG-gaited CT in order to obtain AAA geometry in diastolic phase of cardiac cycle. Some studies presented results of FEM analysis after applying the full systolic pressure to AAA model [32, 52]. The used systolic pressure is either obtained from the patient or assumed as a value from the range $110-130 \mathrm{~mm} \mathrm{Hg}$. Others used mean arterial pressure (MAP) as a loading force [53, 54]. Both cases may lead to overestimation of wall stress, as the aneurysm is already under diastolic blood pressure.

Another approach is based on numerous techniques of acquiring the non-stressed AAA model before applying actual blood pressure [55-57]. It is achieved by reverse simulation resulting in AAA model at "zero-pressure" circumstances.

The introduction of the haemodynamics of blood flow into simulations was a breakthrough in the analysis [50]. Blood is, in fact, viscous substance and thus, its flow is laminar. It means that there are various velocities of the blood flow, depending on the distance from the vessel wall. The highest velocity occurs in the centre of the vessel and the lowest alongside the wall. The different types of velocity profile were examined by Chandra on the basis of the MRI scans [58]. As a consequence of laminar flow in tortuous vessel and the pulsation of the blood stream, the non-uniform pressure distribution develops on the inner surface of the wall. Haemodynamic load is closer to the real conditions occurring in the aneurysm than the static pressure, and thereby produces more reliable results from FEM analysis. The examples of different kinds of load used in FEM simulations are presented in Table 6.

\section{Shear stress}

In some studies, the lack of application of the shear stress is listed as an experiment limitation. However, the relevance of this parameter is questionable. Shear stress acts along the surface to which it is applied and causes geometrical deformation due to the "pulling" effect. It is intuitive to assume that blood flow affects the AAA wall in such manner. Nevertheless, it has been argued that shear stress may be omitted in computer simulations as it does not affect the wall directly, since most of aneurysms have ILT deposits as an obstacle between wall and blood flow, and the value of possible shear stress is several orders of magnitude lower than pressure induced wall stress [60].

\section{Peak Wall Stress (PWS)}

The rupture of the aneurysm emerges when mechanical stress exceeds strength of the vessel. Thus, assessment of the occurrence of that situation is crucial. Peak wall stress is, in fact, the maximal stress that occurs in aneurysmatic wall during the cardiac cycle. PWS is obtained from FEM analysis which incorporates individualized, geometrical and biomechanical parameters of the aneurysm. It has been proven that PWS is signifi-

Table 6. Mechanical parameters of simulation load

\begin{tabular}{|c|c|c|}
\hline Research & Features & Mathematical parameters \\
\hline Speelman et al. [32] & $\begin{array}{l}\text { Uniform pressure applied to the } \\
\text { inner surface of the models }\end{array}$ & $16 \mathrm{kPa}$ \\
\hline Gasser et al. [53] & $\begin{array}{l}\text { Uniform pressure applied to the } \\
\text { inner surface of the models }\end{array}$ & $\begin{array}{l}M A P=p_{d}+I / 3\left(p_{s}-p_{d}\right) \\
p_{s}-\text { systolic pressure } \\
p_{d}-\text { diastolic pressure }\end{array}$ \\
\hline Scotti et al. [59] & Fluid flow & $\begin{array}{c}\nabla \cdot v=0 \\
\rho \frac{\partial v}{\partial t}+\rho v \cdot \nabla v-\nabla \cdot \tau=f^{B} \\
\tau=-p \delta_{i j}+2 \mu \varepsilon_{i j} \\
M=3.5 \mathrm{cP}, \rho=1.05 \mathrm{~g} / \mathrm{cm}^{3}\end{array}$ \\
\hline
\end{tabular}

Where $\rho$ is the fluid density, $\mathrm{v}$ is the velocity vector, $\tau$ the stress tensor, $\mathrm{p}$ is the fluid pressure, $\delta_{\mathrm{ij}}$ is the Kronecker delta, $\mu$ is the dynamic viscosity of the fluid, and $\varepsilon_{\mathrm{ij}}$ is the strain rate and $f^{B}$ is the body force 
cantly higher in the AAAs near the time of the rupture in contrast to electively repaired aneurysms $[17,40]$. The positive correlation between the PWS and the localization of rupture has been proven [4I]. In consequence, PWS seems to be more accurate predictor of the AAA rupture than simple geometric based indicators $[7,8$, 61]. The impacts of numerous biomechanical factors on PWS were studied. Georgakarakos showed that internal tortuosity affect the value and localization of PWS. It was highlighted that the tortuosity depends on the ILT distribution. Thus, the ILT, as geometrical component of AAA, must be included into the aneurysm model [62].

It is important to notice that PWS estimates maximal wall stress and does not take into account information regarding wall strength. The latter varies amongst patients and even within the given aneurysm. Thus, PWS might be treated only as indicator of the weakest areas of whole vessel, not as a measure of rupture risk likelihood.

\section{Conclusions}

In this paper, the important parameters of the FEM analysis were presented. Since the Law of Laplace and the consequent threshold of $55 \mathrm{~mm}$ of the AAA diameter as a cut off value for the surgical interventionhave limitations, there is a need for the development of new predictors of rupture risk. Numerous factors and their impact on the aneurysmswhere studied resulting in different approaches to FEM analysis. Nevertheless, the predominance of detailed biomechanical investigation was proven. As it was stated, geometrical parameters play essential role in computer simulations of AAA behaviour. That is why development in imaging techniques resulting in acquisition the precise AAA shape is desirable. It is worth noticing that all the mentioned mechanical parameters are only mathematical models which do not accurately reflect the behaviour of complex aneurysm. Further investigation on that subject would be highly beneficial. Nevertheless, the results obtained from the computer simulations are far more reliable predictor of AAA rupture than the conventional diameter criterion. Thus FEM analysis should be continually improved and ultimately introduced into the clinical practice.

\section{References}

I. Johnston KW, Rutherford RB, Tilson MD et al (1991) Suggested standards for reporting on arterial aneurysms. J Vasc Surg; 13: 452-458.

2. Żukowski M, Biernawska J, Kotfis K et al (2013) Perioperative factors influencing the mortality of elective abdominal aorta aneurysm repair. Acta Angiol; 19: I-8.
3. Fillinger M (2007) Who should we operate on and how do we decide: predicting rupture and survival in patients with aortic aneurysm. Semin Vasc Surg; 20: 121-127.

4. Nicholls SC, Gardner JB, Meissner MH et al (1998) Rupture in small abdominal aortic aneurysms. J Vasc Surg; 28: 884-888.

5. Thompson SG, Brown LC, Sweeting MJ et al (2013) Systematic review and meta-analysis of the growth and rupture rates of small abdominal aortic aneurysms: implications for surveillance intervals and their cost-effectiveness. Health Technol Assess; 17: $1-118$.

6. Powell JT, Gotensparre SM, Sweeting MJ et al (20II) Rupture rates of small abdominal aortic aneurysms: A systematic review of the literature. European Journal of Vascular and Endovascular Surgery; 4I: 2-10.

7. Hua J, Mower WR (200I) Simple geometric characteristics fail to reliably predict abdominal aortic aneurysm wall stresses. J Vasc Surg; 34: 308-315.

8. Sacks MS, Vorp DA, Raghavan ML et al (1999) In vivo three-dimensional surface geometry of abdominal aortic aneurysms. Ann Biomed Eng; 27: 469-479.

9. Schriefl AJ, Zeindlinger G, Pierce DM et al (20/2) Determination of the layer-specific distributed collagen fibre orientations in human thoracic and abdominal aortas and common iliac arteries. J R Soc Interface; 9: 1275-1286.

10. Di Martino ES, Bohra A, Vande Geest JP et al (2006) Biomechanical properties of ruptured versus electively repaired abdominal aortic aneurysm wall tissue. J Vasc Surg; 43: 570-576.

II. Raghavan ML, Kratzberg J, Castro de Tolosa EM et al (2006) Regional distribution of wall thickness and failure properties of human abdominal aortic aneurysm. J Biomech; 39: 3010-3016.

12. Reeps C, Maier A, Pelisek J et al (2013) Measuring and modeling patient-specific distributions of material properties in abdominal aortic aneurysm wall. Biomech Model Mechanobiol; 12: 7I7-733.

13. Mohammad NF, Nassriq MN, Saidatul A (2009) Analysis of blood flow, pressure and velocity in a stented abdominal aortic aneurysm model. Proceedings of International Conference on Applications and Design in Mechanical Engineering (iCADME).

14. Raut SS, Jana A, De Oliveira $V$ et al (2013) The importance of patient-specific regionally varying wall thickness in abdominal aortic aneurysm biomechanics. J Biomech Eng; 135: 0810101$-08101010$.

15. Raghavan ML, Vorp DA, Federle MP et al (2000) Wall stress distribution on three-dimensionally reconstructed models of human abdominal aortic aneurysm. J Vasc Surg; 3: 760-769.

16. Doyle BJ, Cloonan AJ, Walsh MT et al (2010) Identification of rupture locations in patient-specific abdominal aortic aneurysms using experimental and computational techniques. J Biomech; 43: 1408-1416.

17. Fillinger MF, Marra SP, Raghavan ML et al (2003) Prediction of rupture risk in abdominal aortic aneurysm during observation: Wall stress versus diameter. J Vasc Surg; 37: 724-732.

18. Venkatasubramaniam AK, Fagan MJ, Mehta T et al (2004) A comparative study of aortic wall stress using finite element analysis for ruptured and non-ruptured abdominal aortic aneurysms. Eur J Vasc Endovasc Surg; 28: 168-176.

19. Mower WR, Quinones WJ, Gambhir SS (1997) Effect of intraluminal thrombus on abdominal aortic aneurysm wall stress. J Vasc Surg; 26: 602-608. 
20. Wang DHJ, Makaroun MS, Webster MW et al (2002) Effect of intraluminal thrombus on wall stress in patient-specific models of abdominal aortic aneurysm. J Vasc Surg; 36: 598-604.

21. Thubrikar MJ, Robicsek F, Labrosse M et al (2003) Effect of thrombus on abdominal aortic aneurysm wall dilation and stress. J Cardiovasc Surg; 44: 67-77.

22. Inzoli F, Boschetti F, Zappa M et al (1993) Biomechanical factors in abdominal aortic aneurysm rupture. Eur J Vasc Surg; 7: 667-674.

23. Vorp DA, Lee PC, Wang DHJ et al (200I) Association of intraluminal thrombus in abdominal aortic aneurysm with local hypoxia and wall weakening. J Vasc Surg; 34: 29I-299.

24. Kazi M, Thyberg J, Religa $P$ et al (2003) Influence of intraluminal thrombus on structural and cellular composition of abdominal aortic aneurysm wall. J Vasc Surg; 38: 1283-1292.

25. Thompson JF, Mullee MA, Bell PR et al (1996) Intraoperative heparinisation, blood loss and myocardial infarction during aortic aneurysm surgery: a Joint Vascular Research Group study. Eur J Vasc Endovasc Surg; 12: 86-90.

26. Newman KM, Jean-Claude J, Li H et al (1994) Cellular localization of matrix metalloproteinases in the abdominal aortic aneurysm wall. J Vasc Surg; 20: 814-820.

27. Campbell EJ, Wald MS (1983) Hypoxic injury to human alveolar macrophages accelerates release of previously bound neutrophil elastase. Am Rev Respir Dis; 127: 631-635.

28. Kowalewski R, Panek B, Pałka J et al (2007) Assessment of some of the factors involved in collagen metabolism in the abdominal aortic aneurysm wall. Acta Angiol; 13: 56-64.

29. Herrick SE, Ireland GW, Simon D et al (1996) Venous ulcer fibroblasts compared with normal fibroblasts show differences in collagen but not fibronectin production under both normal and hypoxic conditions. J Invest Dermatol; 106: 187-193.

30. Steinbrech DS, Longaker MT, Mehrara BJ et al (1999) Fibroblast response to hypoxia: the relationship between angiogenesis and matrix regulation. J Surg Res; 84: 127-133.

3I. Stenbaek J, Kalin B, Swedenborg J (2000) Growth of thrombus may be a better predictor of rupture than diameter in patients with abdominal aortic aneurysms. Eur J Vasc Endovasc Surg; 20: 466-469.

32. Speelman L, Bohra A, Bosboom EMH et al (2007) Effects of Wall Calcifications in Patient-Specific Wall Stress Analyses of Abdominal Aortic Aneurysms. J Biomech Eng; 129: 105-109.

33. Li ZY, U-King-Im J, Tang TY et al (2008) Impact of calcification and intraluminal thrombus on the computed wall stresses of abdominal aortic aneurysm. J Vasc Surg; 47: 928-935.

34. Maier A, Gee MW, Reeps $C$ et al (2010) Impact of calcifications on patient-specific wall stress analysis of abdominal aortic aneurysms. Biomech Model Mechanobiol; 9: 5II-52I.

35. Fillinger MF, Racusin J, Baker RK et al (2004) Anatomic characteristics of ruptured abdominal aortic aneurysm on conventional CT scans: Implications for rupture risk. J Vasc Surg; 39: 1243-1252.

36. Elger DF, Blackketter DM, Budwig RS et al (1996) The Influence of Shape on the Stresses in Model Abdominal Aortic Aneurysms. J Biomech Eng; II8: 326-332.

37. Raghavan ML, Vorp DA (2000) Toward a biomechanical tool to evaluate rupture potential of abdominal aortic aneurysm: Identification of a finite strain constitutive model and evaluation of its applicability. J Biomech; 33: 475-482.
38. Thubrikar MJ, Al-Soudi J, Robicsek F (200I) Wall stress studies of abdominal aortic aneurysm in a clinical model. Ann Vasc Surg; 15: 355-366.

39. Scotti CM, Shkolnik AD, Muluk SC et al (2005) Fluid-structure interaction in abdominal aortic aneurysms: effects of asymmetry and wall thickness. Biomed Eng Online; 4: 64-86.

40. Fillinger MF, Raghavan ML, Marra SP et al (2002) In vivo analysis of mechanical wall stress and abdominal aortic aneurysm rupture risk. J Vasc Surg; 36: 589-597.

4I. Venkatasubramaniam AK, Fagan MJ, Mehta T et al (2004) A comparative study of aortic wall stress using finite element analysis for ruptured and non-ruptured abdominal aortic aneurysms. Eur J Vasc Endovasc Surg; 28: 168-176.

42. O'Leary SA, Healey DA, Kavanagh EG et al (2014) The biaxial biomechanical behavior of abdominal aortic aneurysm tissue. Ann Biomed Eng; 42: 2440-2450.

43. Vande Geest JP, Sacks MS, Vorp DA (2006) The effects of aneurysm on the biaxial mechanical behavior of human abdominal aorta. J Biomech; 39: 1324-1334.

44. Wang DH, Makaroun M, Webster MW (200I) Mechanical properties and microstructure of intraluminal thrombus from abdominal aortic aneurysm. J Biomech Eng; 123: 536-539.

45. Van Dam EA, Dams SD, Peters GWM et al (2008) Non-linear viscoelastic behavior of abdominal aortic aneurysm thrombus. Biomech Model Mechanobiol; 7: 127-137.

46. Ayyalasomayajula A, Vande Geest JP, Simon BR (2010) Porohyperelastic finite element modeling of abdominal aortic aneurysms. J Biomech Eng; I32: 104502.

47. Polzer S, Bursa J (2010) Poroelastic model of intraluminal thrombus in FEA of aortic aneurysm. IFMBE Proceedings; 31: 763-767.

48. Marra SP, Daghlian CP, Fillinger MF et al (2006) Elemental composition, morphology and mechanical properties of calcified deposits obtained from abdominal aortic aneurysms. Acta Biomater; 2: 515-520.

49. Loree HM, Grodzinsky AJ, Park SY et al (1994) Static circumferential tangential modulus of human atherosclerotic tissue. J Biomech; 27: 195-204.

50. Di Martino ES, Guadagni G, Fumero A et al (200I) Fluid-structure interaction within realistic three-dimensional models of the aneurysmatic aorta as a guidance to assess the risk of rupture of the aneurysm. Med Eng Phys; 23: 647-655.

5I. Vande Geest JP, Sacks MS, Vorp DA (2006) A planar biaxial constitutive relation for the luminal layer of intra-luminal thrombus in abdominal aortic aneurysms. J Biomech; 39: 2347-2354.

52. Huang $\mathrm{H}$, Virmani R, Younis $\mathrm{H}$ et al (200I) The impact of calcification on the biomechanical stability of atherosclerotic plaques. Circulation; 103: 105I-1056.

53. Gasser TC, Auer M, Labruto F et al (2010) Biomechanical rupture risk assessment of abdominal aortic aneurysms: Model complexity versus predictability of finite element simulations. Eur J Vasc Endovasc Surg; 40: 176-185.

54. Larsson E, Labruto F, Gasser TC et al (201 I) Analysis of aortic wall stress and rupture risk in patients with abdominal aortic aneurysm with a gender perspective. J Vasc Surg; 54: 295-299.

55. de Putter S, Wolters BJBM, Rutten MCM et al (2007) Patient-specific initial wall stress in abdominal aortic aneurysms with a backward incremental method. J Biomech; 40: 108I-1090.

56. Raghavan ML, Ma B, Fillinger MF (2006) Non-invasive determination of zero-pressure geometry of arterial aneurysms. Ann Biomed Eng; 34: 1414-1419. 
57. Speelman L, Bosboom EMH, Schurink GWH et al (2009) Initial stress and nonlinear material behavior in patient-specific AAA wall stress analysis. J Biomech; 42: 1713-1719.

58. Chandra S, Raut SS, Jana A et al (2013) Fluid-structure interaction modeling of abdominal aortic aneurysms: the impact of patient-specific inflow conditions and fluid/solid coupling. J Biomech Eng; 135: 81001.

59. Scotti CM, Finol EA (2007) Compliant biomechanics of abdominal aortic aneurysms: A fluid-structure interaction study. Comput Struct; 85: 1097-I I I3.
60. Peattie RA, Riehle TJ, Bluth El (2004) Pulsatile flow in fusiform models of abdominal aortic aneurysms: flow fields, velocity patterns and flow-induced wall stresses. J Biomech Eng; 126: 438-446.

6I. Fillinger MF, Marra SP, Raghavan ML et al (2003) Prediction of rupture risk in abdominal aortic aneurysm during observation: Wall stress versus diameter. J Vasc Surg; 37: 724-732.

62. Georgakarakos E, loannou CV, Kamarianakis $Y$ et al (2010) The role of geometric parameters in the prediction of abdominal aortic aneurysm wall stress. Eur J Vasc Endovasc Surg; 39: 42-48. 